\title{
Generation of complex concentration profiles in microchannels in a logarithmically small number of steps
}

\author{
Kyle Campbell and Alex Groisman*
}

\author{
Received 18th July 2006, Accepted 6th November 2006 \\ First published as an Advance Article on the web 27th November 2006 \\ DOI: $10.1039 / \mathrm{b} 610011 \mathrm{~b}$
}

We describe the principles of design and the architecture of planar microfluidic networks producing concentration gradients with the shape of any given monotonic function. Each microfluidic network is fed by two separate source solutions and delivers to its outlet a set of $N$ solutions that all differ in concentration. Inside the network, the source solutions flow through a series of $k=\log _{2}(N-1)$ stages, where they are repeatedly split and mixed. Streams of the solutions emerging from the network are combined to create a single stream with the desired shape of the concentration profile across the direction of flow. To demonstrate the functionality of the proposed architecture, we have built and tested three networks with $k=4$ and $N=17$ that generate an exponential concentration profile, a linear profile, and a profile with a shape of two fused branches of a parabola.

\section{Introduction}

The engineering of controlled liquid environments with gradients of concentration of relevant substances is important for various chemical and biological applications, in particular, for the study of chemotaxis. Reasonably stable gradient profiles are created in simple chemotaxis chambers designed by Zigmond ${ }^{1}$ and by Dunn et al. ${ }^{2}$ that consist of two large wells connected by a narrow channel. A gradient profile is generated by the molecular diffusion through the channel, from the well loaded with a solution with a high concentration of the chemoattractant (source) to the well loaded with a low concentration solution or a plain buffer (sink). This method of gradient generation has the drawbacks of limited control of the profile shape, long time required for the gradient to build up, and eventual deterioration of the gradient due to depletion of the source and contamination of the sink.

Truly stable concentration profiles can be created by using solutions with different concentrations flowing at a constant speed through a microscopic channel. Since the microscopic flows are generally laminar and stable, a state of dynamic equilibrium is reached in the channel-the diffusive flux driven by a concentration gradient across the flow is balanced by constant replenishment of the solutions. The simplest implementation of this approach is with two source solutions injected into a microchannel side by side. While useful for some applications, ${ }^{3,4}$ this arrangement has multiple shortcomings. The concentration profile across the flow always has a characteristic sigmoidal shape, ${ }^{5}$ which may not be optimal for the desired application, and creating a gradient profile of sufficient width may require an impractically long channel. In addition, the shape of the profile depends on the position along the channel and on the flow speed.

Department of Physics, University of California, San Diego, 9500 Gilman Drive, La Jolla, CA, 92093, USA.E-mail: agroisman@ucsd.edu
These problems were successfully resolved in the devices introduced by Whitesides et al. ${ }^{6,7}$ Variations of these devices have been used for a variety of biological applications, including experiments on chemotaxis of human neutrophils, ${ }^{8-12}$ cancer cells ${ }^{13}$ and amoebas; ${ }^{14}$ on culturing of cells at different medium conditions; and on cell dose response. ${ }^{15-17}$ Among other applications there have been titration, ${ }^{18}$ differential patterning of surfaces, ${ }^{19-21}$ fabrication of materials with spatially modified properties, ${ }^{22,23}$ and on-chip adjustment of refractive index. ${ }^{24} \mathrm{~A}$ key part of the devices is a gradientmaking network of microchannels that typically has a recognizable pyramidal shape. ${ }^{6,7}$ In its basic configuration, the network is supplied with two source solutions and delivers to its outlet a set of $N$ solutions with a linear sequence of concentrations. Inside the network the source solutions flow through a series of stages, where they are repeatedly split and mixed in different proportions. At the network outlet, streams of the $N$ solutions merge into a single stream with linear variation of concentration across the direction of flow.

The gradient-making network by Whitesides et al. can be readily adapted to generate concentration profiles with shapes of power functions and polynomials. ${ }^{6,7,25,26}$ However, generation of a polynomial of a power $m$ requires $m+1$ separate source solutions, becoming impractical at large $m$, and there is no straightforward way to generate non-polynomial shapes such as an exponent. In addition, the number of splitting-andmixing stages linearly increases with $N$, and while large $N$ is beneficial for the accuracy and stability of the concentration profile, in most practical implementations of the network $N \leqslant$ 10. An alternative gradient-making network allowing generation of complex concentration profiles has been recently proposed by Toner et $a l .{ }^{27}$ However, it was only shown to be operational at very low flow rates $\left(20 \mu \mathrm{m} \mathrm{s}^{-1}\right)$, and just as in the network in ref. 6, the number of steps of splitting and mixing was linearly increasing with $N$.

Here we present an alternative architecture for microfludic gradient-making networks that has two advantages over the 
original design by Whitesides et al ${ }^{6,7}$ First, it allows generating a monotonic concentration profile of any given shape. Second, a logarithmically small number of splitting-and-mixing stages $\left(\sim \log _{2} N\right)$ is required to produce a set of $N$ solutions with different concentrations, making the network more compact and substantially increasing the practical limit on $N$. We have designed, fabricated and tested three microfluidic devices that produce an exponential concentration profile, a linear profile, and a profile with a shape of two fused branches of a parabola.

\section{Experimental}

\section{Microfabrication}

Each of the three microfluidic devices described in this work (Fig. 1) has two inlets, one outlet and is made of a single cast of polydimethylsiloxane (PDMS; Sylgard 182, Dow Corning, Midland, MI) bonded to a microscope cover glass. A master mold to cast the PDMS chips was fabricated by spin-coating a 40 micron layer of negative photoresist (SU-8 2015, Microchem, Newton, MA) onto a 5" silicon wafer and exposing it to UV-light through a specially designed photomask with a resolution of 40,000 dpi. PDMS prepolymer was poured onto the master mold, degassed and cured by baking in an $80{ }^{\circ} \mathrm{C}$ oven for $1.5 \mathrm{~h}$. Individual chips were cut from the cured PDMS, and inlet and outlet holes were punched with a 20 gauge luer-stub. The chips were soaked in $0.01 \mathrm{M} \mathrm{HCl}$ at $80{ }^{\circ} \mathrm{C}$ for $1 \mathrm{~h}$ to make the surface more hydrophilic. The devices were completed by attaching \#1.5 cover glasses to the patterned surfaces of the PDMS chips and baking them at $80{ }^{\circ} \mathrm{C}$ overnight to promote adhesion between the PDMS and the glass.

\section{Flow control}

The working liquids were kept in $60 \mathrm{cc}$ plastic syringes, which were held upright with the luer connector at the bottom and were open to the atmosphere at the top. Each syringe was connected to a microfluidic device through a blunt luer needle (gauge 23), a long piece of Tygon tubing with an internal diameter of $0.5 \mathrm{~mm}$, and a short piece of hypodermic steel tubing inserted into an inlet or outlet of the device. The flow in the microchannels was driven by setting a difference in pressure between the inlets and the outlet, $P_{\mathrm{d}}$. The differential pressure was generated hydrostatically by attaching the two syringes connected to the inlets to separate moving stages sliding along a vertical rail with a precise ruler. ${ }^{28}$ The syringe connected to the outlet was held at a constant lower level. The difference in the levels of liquid between the syringes connected to the inlets and to the outlet was measured with a precision of about $0.2 \mathrm{~mm}$, corresponding to $2 \mathrm{~Pa}$ in pressure.

\section{Chemicals}

To visualize concentration profiles in microchannels we used solutions of fluorescein isothiocyanate, FITC, (Sigma, St. Louis, MO) with a diffusion coefficient $D=5 \times 10^{-6} \mathrm{~cm}^{2} \mathrm{~s}^{-1}$. To stabilize its fluorescence level, FITC was dissolved in a $\mathrm{pH}=7.5$ phosphate buffer.

\section{Microscopy and measurements}

Microfluidic devices were mounted on an inverted fluorescence microscope Nikon TE2000U, which was equipped with a cooled digital CCD camera, Spot RT-SE6 (Diagnostic Instruments, Sterling Heights, MI), and an electronic shutter in the fluorescence illumination light train. The shutter was normally closed to prevent excessive photobleaching; its opening was synchronized with taking fluorescence images. The concentration of FITC in microchannels in a specific area of a microfludic device was evaluated by measuring the intensity of light at that location in a fluorescence image. Before the fluorescent light intensity was measured, the image was pre-processed in two steps. First, the background light
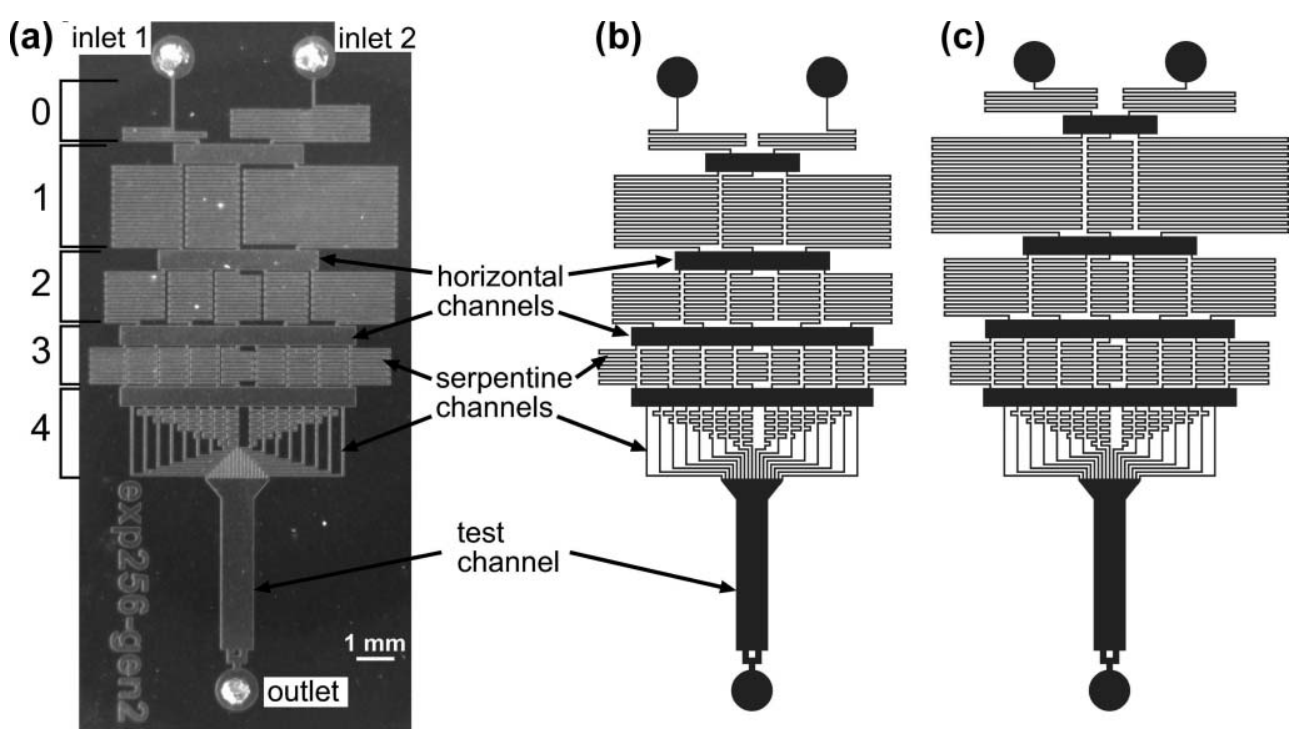

Fig. 1 Microfluidic devices. (a) Micrograph of the microfludic device generating an exponential concentration profile. (b), (c) Drawings of the microfluidic devices generating a linear and double-parabolic concentration profile, respectively. Numbers on the left indicate individual stages of splitting-and-mixing. 
level, corresponding to known non-fluorescent areas, was subtracted from the image. Second, the image was normalized with a distribution of fluorescent light from a slab of a uniformly fluorescent material at the same illumination and imaging condition. This normalization (flat-field correction) was performed to compensate for non-uniformity in the fluorescence illumination, light collection and sensitivity of the CCD array. To test for linearity of the dependence of the fluorescence intensity on the FITC concentration, we took images of microchannels with different known concentration of FITC inside. In the working range of $0-15 \mathrm{ppm}$, the dependence was found to be linear within $\sim 1 \%$. Maximal flow velocity in channels, $v_{\max }$, at various driving pressures, $P_{\mathrm{d}}$, was evaluated by seeding solutions fed into the device with $4.6 \mu \mathrm{m}$ fluorescent particles (Interfacial Dynamics Corp, Portland, OR) and measuring the extension of the longest streaklines produced by the particles. The mean flow velocity, $\bar{v}$, in the $50 \times 40 \mu \mathrm{m}$ serpentine channels was computed as $\bar{v}=0.48 v_{\max }$, with the ratio between $\bar{v}$ and $v_{\max }$ calculated using equations from ref. 29.

\section{Design and operation of the microfluidic devices}

Similarly to the devices introduced by Whitesides et al., ${ }^{6,7}$ each of the microfluidic devices described in this paper consists of two parts: a gradient-making network and a test channel (wide vertical channel in Fig. 1(a), (b) and (c)). The gradient-making network is fed by two source solutions with different concentrations injected into the device through separate inlets, and it generates $N$ solutions of different concentrations. These latter solutions are directed into the test channel through $N$ separate narrow channels (the lowest row of serpentine channels in Fig. 1). In all three devices described in this paper $N=17$ (Fig. 1). It is convenient to number the serpentine channels from left to right, and designate the concentration in the $n$th serpentine channel as $c_{n}$, where $1 \leqslant n \leqslant N$. The set of $c_{n}$ is constructed to be a discrete representation of the desired concentration profile across the test channel, $c(y)$.

Streams of all solutions injected into the test channel have the same volumetric flux and approximately the same width in the test channel, $\Delta y \approx w / N$, where $w$ is the width of the test channel. Exceptions are the two streams at the edges, which are somewhat wider because the flow velocity near the edges of the test channel is lower than in the middle. As the flow advances along the test channel, the initial discontinuity in concentration between adjacent streams gradually disappears due to molecular diffusion. The characteristic diffusion time is $t_{\mathrm{D}}=\Delta y^{2} / D$, corresponding to a characteristic distance $\Delta x=$ $\bar{v} t_{D}$ from the stream merging point at the beginning of the test channel. Here $D$ is the coefficient of diffusion of the solute, and $\bar{v}$ is the mean flow speed. (For the actual test channel, the analysis is somewhat more complicated because of variation of the channel width in the beginning.) At distances $x>\Delta x, c(y)$ is a smooth function. Molecular diffusion across the test channel also levels off the non-uniformity of $c(y)$ on the scale of the whole channel, making $c(y)$ increasingly different from its desired shape as $x$ and the residence time in the flow increase. The characteristic time of this unwanted leveling off can be estimated as $w^{2} / D=(N \Delta y)^{2} / D=N^{2} t_{\mathrm{D}}$, corresponding to a distance $N^{2} \Delta x$ from the beginning. These simple arguments show that a suitable working range of $x$, at which the distribution $c(y)$ is both smooth and closely matching the set of $c_{n}$, is given by $\Delta x<x \ll N^{2} \Delta x$. Furthermore, at $\Delta x<x \ll$ $N^{2} \Delta x$, the concentration profile has little sensitivity to variations of $\bar{v}$. Therefore, it is essential that $N$ is sufficiently large, $N^{2} \gg 1$, for the device to operate well. In addition to providing a long working region in the test channel, a large value of $N$ also allows accurate definition of $c(y)$ by the discrete set of $c_{n}$. The latter is especially important for nonlinear $c(y)$, because the molecular diffusion between the adjacent streams in the test channel tends to reduce the curvature of the concentration profile.

The proposed gradient-making networks have a layout similar to the networks introduced by Whitesides et al.: ${ }^{6,7}$ they consist of consecutive stages, each comprised of a wide horizontal channel and a set of narrow serpentine channels downstream from it (Fig. 1). (The latter channels are called vertical channels in ref. 6 and 7). The horizontal channels distribute the flow emerging from the upstream serpentine channels over a larger number of the downstream serpentine channels. In addition, due to low flow resistance of the horizontal channels, all serpentine channels belonging to the same stage have equal pressures at their inlets and equal pressures at their outlets. The function of the serpentine channels is to provide diffusive mixing of solutions injected into them and to generate a homogeneous solution of an intermediate concentration, which is fed into a downstream horizontal channel. The diffusive mixing is facilitated by the small width and large length of the serpentine channels, resulting in small characteristic diffusion time and large liquid residence time, respectively. Volumetric flux through a channel, $q=\Delta P / R$, is defined by the difference in pressure between the inlet and the outlet, $\Delta P$, and by the channel flow resistance, $R$. The flow resistance is proportional to the channel contour length, $R=\kappa L$, where $\kappa$ is the same for all serpentine channels, as they all have the same width and depth, $w_{\mathrm{s}}=50 \mu \mathrm{m}$ and $h=40 \mu \mathrm{m}$, respectively.

The gradient-making networks in the devices shown in Fig. 1 differ from those in ref. 6 and 7 in two important regards. First, serpentine channels belonging to the same stage have different lengths, $L$, leading to different $R$ and different $q$ through the channels. Variability of $q$ makes it possible to mix solutions in individually adjustable proportions and to generate concentration profiles with a variety of shapes. Second, the stream from an individual serpentine channel of the $k$ th stage is split between 3 serpentine channels of the $(k+1)$ th stage (Fig. 2 and 3$)$, as compared to 2 channels of the $(k+1)$ th stage in ref. 6 and 7. (An exception are streams from the channels at the edges, which are split between 2 channels of the $(k+1)$ th stage just as in ref. 6 and 7). The proposed gradient-making networks are built so that each oddnumbered serpentine channel of the $(k+1)$ th stage is fed by a single channel of the $k$ th stage and thus carries solution of the same concentration (Fig. 2 and 3). Each even-numbered serpentine channel of the $(k+1)$ th stage is fed by two adjacent channels of the $k$ th stage, and a solution with an intermediate concentration is generated in it. The numbers of solutions with different concentrations within the $k$ th and $(k+1)$ th stages, $N_{k}$ 


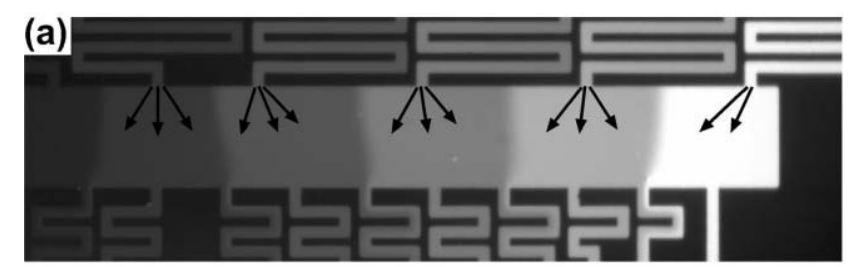

(b)

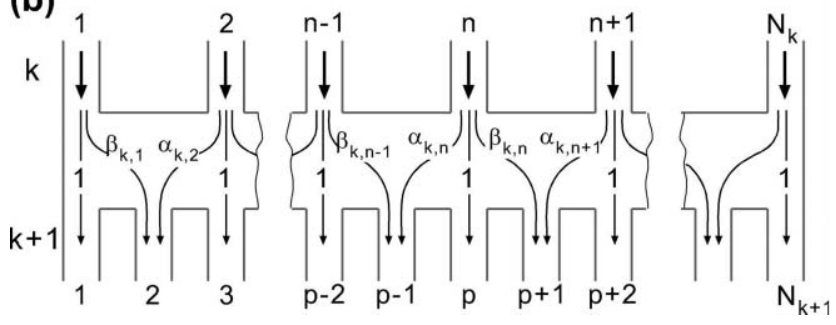

Fig. 2 Splitting and redistribution of flux in a horizontal channel. (a) Fluorescence micrograph of the right hand side of the horizontal channel of the 4th stage in the gradient-making network generating an exponential profile, with serpentine channel of the $3 \mathrm{rd}$ and 4 th stage at the top and bottom, respectively. The grayscale look-up table is nonlinearly adjusted for better visibility. Arrows indicate the direction of flow. (b) Schematic diagram showing splitting and redistribution of flux between serpentine channels of the $k$ th and $(k+1)$ th stages and illustrating the meaning of the coefficients $\alpha$ and $\beta$. Serpentine channels of the $k$ th and $(k+1)$ th stages are shown as vertical channels at the top and bottom, respectively, and are numbered from 1 to $N_{k}$ and from 1 to $N_{k+1}$, respectively. Coefficients at the arrows show portions of flux in the channel of the $(k+1)$ th stage supplied by the channel of the $k$ th stage.

and $N_{k+1}$, are connected by a recurrence relation, $N_{k+1}=$ $N_{k}+\left(N_{k}-1\right)$. Given $N_{1}=3$, we derive $N_{k}=2^{k}+1$ and $k=$ $\log _{2}\left(N_{k}-1\right)$, meaning that the number of stages in the proposed gradient-making networks increases only logarithmically with the number of distinct concentrations at the network outlet.

Construction of a gradient-making network of the proposed architecture begins with the definition of the desired shape of the concentration profile, $c(y)$, and the number of stages in the network. All three networks described here have 4 stages, corresponding to $N=N_{4}=2^{4}+1=17$ serpentine channels in the last stage. To keep the notation more compact, we will designate the $n$th serpentine channel (as counted from the left) of the $k$ th stage as channel $<k, n>$ (Fig. 2b). The concentration and volumetric flux in channel $<k, n>$ will be designated as $c_{k, n}$ and $q_{k, n}$, respectively. The resistance and length of channel $\left\langle k, n>\right.$ will be designated as $R_{k, n}$ and $L_{k, n}$, respectively. We will next consider in detail the construction of a network generating an exponential profile with a ratio of 256 between the highest and lowest concentration (Fig. 3).

The concentrations in the serpentine channels of the last (4th) stage are chosen as a discrete set approximating the desired exponential profile, $c_{n}=c_{4, n}=[1, \sqrt{2}, 2,2 \sqrt{2}, 4,4 \sqrt{2}, 8, \ldots, 128,128 \sqrt{2}, 256]$ (in relative units). The concentrations in successive channels differ by a constant factor of $\sqrt{2}$, so the sequence $c_{n}$ is a geometric progression. The condition that each odd-numbered channel of the $(k+1)$ th stage be fed by a single channel from the $k$ th stage uniquely defines the set of concentration in the serpentine channels
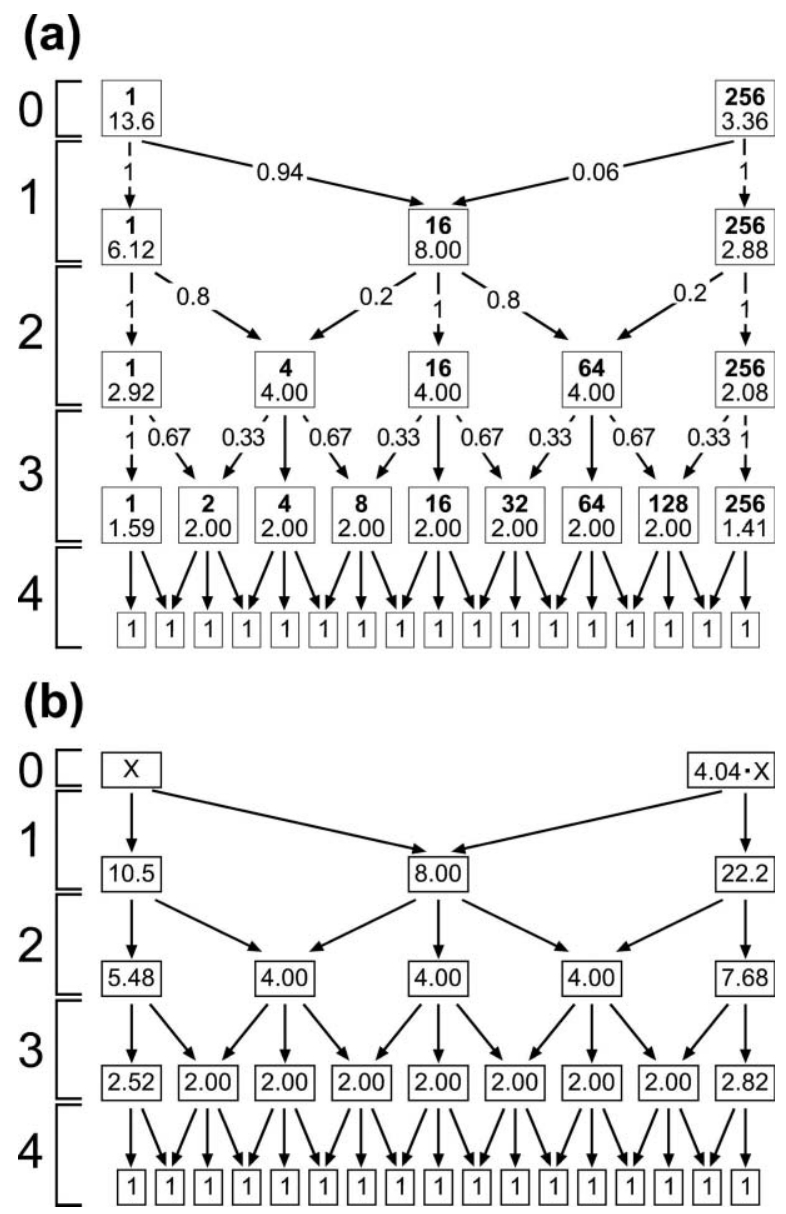

Fig. 3 Schematic diagrams illustrating the construction of a gradientmaking network generating an exponential concentration profile with the concentration increasing by a factor of 256 from left to right. Boxes represent individual serpentine channels ( $c f$. Fig. 1). Arrows show redistribution of flux in the horizontal channels ( $c f$. Fig. 1 and 2). Numbers on the left indicate individual stages of splitting-and-mixing. (a) Numbers in the boxes show the concentrations (upper row in bold; not shown for the 4th stage) and volumetric fluxes (lower row) in the serpentine channels (both in relative units). Numbers on the arrows are portions of the flux in the channels at the arrow heads supplied by the channels at the arrow tails: coefficients $\alpha_{k, n}$ and $\beta_{k, n}$ for arrows pointing left and right, respectively, and 1 for vertical arrows ( $c f$. Fig. 2(b)). (b) Numbers in the boxes indicate lengths (and resistances) of the channels in relative units, as calculated from the equations $L_{k, n} / L_{k, m}=q_{k, m} / q_{k, n}$ and $L_{1,2}=2 L_{2,3}=4 L_{3,5}=8 L_{4,9}$.

of the 3rd stage: $c_{3, n}=[1,2,4,8,16,32,64,128,256]$. Applied consecutively from bottom to top, the same condition defines the concentrations in the serpentine channels of the 2nd and 1st stages, as well as in the two channels connecting the inlets with the horizontal channel of the 1 st stage, $c_{0,1}=1$ and $c_{0,2}=256$ (Fig. 3(a)). We notice that $c_{0,1}$ and $c_{0,2}$ are the concentrations of the solutions injected into the inlets 1 and 2, respectively.

Volumetric fluxes in serpentine channels of the $k$ th stage are specified by concentrations in channels of the $k$ th and $(k+1)$ th stage and fluxes in channels of the $(k+1)$ th stage (Fig. 2(b) and $3(\mathrm{a})$ ). If channel $<k, n>$ is neither the first nor the last of the $k$ th stage, it supplies flux to three channels of the $(k+1)$ th stage: $\langle k+1, p-1>,\langle k+1, p>$, and $\langle k+1, p+1>$, where 
$p=2 n-1$ (Fig. 2(b) and 3(a)). Channel $<k, n>$ is the sole source of flux supplied to channel $<k+1, p>$ (vertical arrows in Figs. 2(b) and 3(a)). Therefore, the flux in channel $<k, n>$ is calculated as

$$
q_{k, n}=\alpha_{k, n} q_{k+1, p-1}+q_{k+1, p}+\beta_{k, n} q_{k+1, p+1}
$$

. Here, $\alpha_{k, n}$ and $\beta_{k, n}$ are portions of the volumetric fluxes in channels $<k+1, p-1>$ and $<k+1, p+1>$, respectively, which are supplied by channel $<k, n>$ (arrows pointing left and right, respectively, in Figs. 2(b) and 3(a)); $0 \leqslant k \leqslant 3$ and $1 \leqslant n \leqslant 2^{k}+1$. Once solutions fed to channel $<k+1, p-1>$ (from $<k, n>$ and $<k, n-1>$ ) and to channel $<k+1, p+1>$ (from $<k, n>$ and $<k, n+1>$ ) are mixed, the concentrations in these two channels become $c_{k+1, p-1}=\alpha_{k, n} c_{k, n}+\beta_{k, n-1} c_{k, n-1}$ and $c_{k+1, p+1}=\beta_{k, n} c_{k, n}+\alpha_{k, n+1} c_{k, n+1}$, respectively (Figs. 2(b) and 3(a)). In order to find $\alpha_{k, n}$ and $\beta_{k, n}$, we further notice that $\alpha_{k, n}+\beta_{k, n-1}=1$ and $\beta_{k, n}+\alpha_{k, n+1}=1$ (Figs. 2(b) and 3(a)). Taken together, these equations give $c_{k+1, p-1}=\alpha_{k, n} c_{k, n}+$ $\left(1-\alpha_{k, n}\right) c_{k, n-1}$ and $c_{k+1, p+1}=\beta_{k, n} c_{k, n}+\left(1-\beta_{k, n}\right) c_{k, n+1}$. Finally, we derive

$$
\begin{gathered}
\alpha_{k, n}=\frac{c_{k+1, p-1}-c_{k, n-1}}{c_{k, n}-c_{k, n-1}} \\
\beta_{k, n}=\frac{c_{k+1, p+1}-c_{k, n+1}}{c_{k, n}-c_{k, n+1}}
\end{gathered}
$$

. For the first channel of a stage, the coefficient $\alpha$ is zero, and for the last channel of a stage the coefficient $\beta$ is zero. The coefficients $\alpha_{k, n}$ and $\beta_{k, n}$ are readily calculated from the values of concentrations in the serpentine channels (which are uniquely defined by the concentrations in the channels of the last stage, $c_{n}=c_{4, n}$ ) and then plugged into eqn (1). The system of eqn (1) is closed by specifying the volumetric fluxes in the serpentine channels of the last stage, $q_{4, n}$, which are all equal to 1 in relative units. The values of $q_{k, n}$ are calculated iteratively, stage by stage from bottom to top (Fig. 3(a)).

To illustrate how individual fluxes are computed using eqn (1)-(3), we consider a few examples (Fig. 2(b) and 3(a)). For the leftmost serpentine channel of stage 3 (channel $<3,1>$ ), we have $k=3, n=1, p=2 n-1=1$, and $\alpha=0$. We calculate $\beta_{3,1}=\frac{c_{4,2}-c_{3,2}}{c_{3,1}-c_{3,2}}=\frac{\sqrt{2}-2}{1-2}=2-\sqrt{2} \approx 0.59$, giving $q_{3,1}=q_{4,1}+$ $\beta_{3,1} q_{4,2} \approx 1+0.59 \approx 1.59$. For channel $<3,5>$, we have $p=2 n-$ $1=9$ and calculate $\alpha_{3,5}=\frac{c_{4,8}-c_{3,4}}{c_{3,5}-c_{3,4}}=\frac{8 \sqrt{2}-8}{16-8}=\sqrt{2}-1 \approx 0.41$ and $\quad \beta_{3,5}=\frac{c_{4,10}-c_{3,6}}{c_{3,5}-c_{3,6}}=\frac{16 \sqrt{2}-32}{16-32}=2-\sqrt{2} \approx 0.59, \quad$ giving $q_{3,5}=\alpha_{3,5} q_{4,8}+q_{4,9}+\beta_{3,5} q_{4,10}=\sqrt{2}-1+1+2-\sqrt{2}=2 . \quad$ For channel $<1,3>$, we have $p=5, \beta=0$ and calculate $\alpha_{1,3}=\frac{c_{2,4}-c_{1,2}}{c_{1,3}-c_{1,2}}=\frac{64-16}{256-16}=0.2$, giving $q_{1,3}=\alpha_{1,3} q_{2,6}+q_{2,7} \approx$ $(0.2 \times 4)+2.08=2.88$. We observe (Fig. 3(a)) that all serpentine channels of any given stage have equal fluxes, except for the first and last channels of the stage. This feature is specific to gradientmaking networks generating exponential and linear profiles (Figs. 1(a) and (b)), and is not found in a general case (e.g. Fig. 1(c)).
The calculated values of $q_{k, n}$ are used to select $L_{k, n}$. For all pairs of channels $\langle k, n>$ and $<k, m>$ belonging to the same $k$ th stage, we have an equation

$$
\frac{L_{k, n}}{L_{k, m}}=\frac{q_{k, m}}{q_{k, n}},
$$

setting coefficients of proportionality between the channel lengths. This equation gives a general recipe for building a functional network. Nevertheless, it does not specify the ratios between lengths of serpentine channels belonging to different stages. These ratios and the actual values of $L_{k, n}$ can be rationally selected based on the characteristic time of diffusive mixing in a serpentine channel and the mean flow velocity, $\bar{v}_{k, s}$, in the shortest channel of the stage, $\langle k, s\rangle$, at typical operation conditions of the device. For any given stage, the shortest serpentine channel has the shortest residence time of liquid in it, $t_{k, s}=L_{k, s} / \bar{v}_{k, s} \propto L_{k, s} / q_{k, s} \propto L_{k, s}^{2}$, and $t_{k, s}$ has to be sufficiently long to allow complete diffusive mixing in the channel. In the network under consideration, the shortest serpentine channel of a stage is always the channel in the middle. We further notice (Fig. 3(a)), that the flux in the channel in the middle is multiplied by 2 as the number of the stage is reduced by 1 . Therefore, for consistently thorough diffusive mixing, the lengths of the channels in the middle are to obey the equation $L_{1,2}=2 L_{2,3}=4 L_{3,5}=8 L_{4,9}$, completing eqn (4) and offering a rule for a rational choice of length ratios between channels belonging to different stages (Fig. 3(b)). Finally, we note that since pressures at the device inlets are equal, the ratio of $L_{0,1}$ and $L_{0,2}$ is given by $\frac{L_{0,1}}{L_{0,2}}=\frac{q_{0,2}}{q_{0,1}}$ (Fig. 2(b)). There is no diffusive mixing in the channels of the 0th stage, however. Therefore, $L_{0,1}$ and $L_{0,2}$ do not have to be proportional to the lengths of the serpentine channels of stages 1-4.

The network described above is constructed to generate an exponential profile of a shape $c(y)=\exp (a y)$, where the left and right edges of the test channel correspond to $y=0$ and $y=1$, respectively, and the maximum concentration is 256 in relative units [corresponding to $a=\ln (256)]$. The second gradientmaking network (Fig. 1(b)) is designed to generate a linear concentration profile in the test channel, $c(y)=b y$. A set of the last-stage concentrations approximating this profile is $c_{4, n}=[0$, $1,2,3,4, \ldots, 14,15,16]$ in relative units. The sets of $c_{3, n}, c_{2, n}$, $c_{1, n}$, and $c_{0, n}$ are found following the procedure described above and are used to calculate the values of $\alpha_{k, n}$ and $\beta_{k, n}$ according to eqn (2)-(3). Finally, the system of eqn (1) is solved using the condition of identical fluxes in the serpentine channels of the last stage, $q_{4, n}=1$. The third gradient-making network (Fig. 1(c)) is constructed to produce a profile with a shape of two fused branches of a parabola taken with opposite signs. For brevity we will call it double-parabolic profile. Its functional form is $c(y)=64\left[1-4(y-0.5)^{2}\right]$ for $0 \leqslant y<0.5$ and $c(y)=64\left[1+4(y-0.5)^{2}\right]$ for $0.5 \leqslant y \leqslant 1$, corresponding to a set of the last-stage concentrations $c_{4, n}=[0,15,28,39,48$, $55,60,63,64,65,68,73,80,89,100,113,128]$.

In the design of all three gradient-making networks (Fig. 1), we followed eqn (4) and implemented the relation $L_{1,2}=2 L_{2,3}$ $=4 L_{3,5}=8 L_{4,9}$, with $L_{1,2} \approx 31 \mathrm{~mm}$. In the networks generating exponential and linear profiles (Fig. 1(a) and (b)), 
the design resulted in identical residence times in the shortest serpentine channels of all stages. In the network generating the double-parabolic profile, the smallest residence time in the network was in channel $<1,2\rangle$. In the two other networks, channel $\langle 1,2\rangle$ was one of the channels with the minimum residence time in the network. Therefore, the maximal flow rate in all three devices was limited by maximal $\bar{v}_{1,2}$ that allowed sufficient time for diffusive mixing in channel $\langle 1,2\rangle$ (Fig. 1). Characteristic diffusion time for an aqueous solution of FITC in a $50 \mu \mathrm{m}$ wide channel can be estimated as $t_{0}=$ $w_{s}^{2} / D=5 \mathrm{~s}$, giving a condition $\bar{v}_{1,2}<\bar{v}_{0}=L_{1,2} / t_{0}=6.2 \mathrm{~mm} \mathrm{~s}^{-1}$ for the proper operation of the gradient-making networks.

\section{Results and discussion}

All three devices were tested with a 15 ppm (by weight) solution of FITC in $\mathrm{pH}=7.5$ phosphate buffer injected into inlet 2. The liquid injected into inlet 1 was plain phosphate buffer for the devices generating the linear and doubleparabolic profiles and a $0.059 \mathrm{ppm}$ solution of FITC (15 ppm solution diluted by a factor of 256 with the buffer) for the device generating the exponential profile. The flow in all three devices was driven at pressure $P_{\mathrm{d}} \approx 7.5 \mathrm{kPa}$. (Because of imperfections in fabrication of the devices, the ratios of resistances of the serpentine channels were somewhat different from their intended values; the pressures at the inlets were individually adjusted to obtain the desired values of $c_{1,2}$ and were different from each other by up to $1.5 \%$.) The volumetric flow rates of liquids injected into inlets 1 and 2, $q_{0,1}$ and $q_{0,2}$, are proportional to the difference in pressure between the inlets and the horizontal channel of the 1st stage. Based on our calculations of flow resistances in the networks, this difference in pressure was estimated at $\sim 27 \%$ of $P_{\mathrm{d}}$ with an absolute value of $\sim 2 \mathrm{kPa}$ for all three networks. Therefore, the level of control of $P_{\mathrm{d}}$ (2 Pa precision) corresponded to controlling $q_{0,1}$ and $q_{0,2}$ within $0.1 \%$.

In order to verify that the flow rate in a device was sufficiently low for thorough diffusive mixing, we measured the distribution of FITC in the stream emerging from channel $<1,2>$. To perform the measurements, we took fluorescence images of the central part of the 2nd stage horizontal channel, where the stream expanded to a width of $\sim 1 \mathrm{~mm}$ allowing for good lateral resolution. For all three devices, the standard deviations of the FITC concentration across the stream were less than $1 \%$ of the mean. The mean flow velocities in the test channel were $0.75,0.75$, and $0.57 \mathrm{~mm} \mathrm{~s}^{-1}$ for the devices generating exponential, linear, and double-parabolic profiles, respectively, which was 29 to 38 times higher than in ref. 27. The mean flow velocity in channel $<1,2>$ was $\bar{v}_{1,2} \approx 6.0 \mathrm{~mm}$ $\mathrm{s}^{-1}$ for all three devices. This value of $\bar{v}_{1,2}$ is consistent with the above estimate for $\bar{v}_{0}$.

Profiles of fluorescence and distributions of FITC across the test channels, $c(y)$, in all three devices closely matched the desired concentration profiles (Fig. 4). Notably, in the device designed to generate an exponential profile (Fig. 4(a) and (d)), $c(y)$ followed an exponential curve over more than two orders of magnitude in concentration. The measured concentration profiles differ from their desired functional shapes in marginal (a)

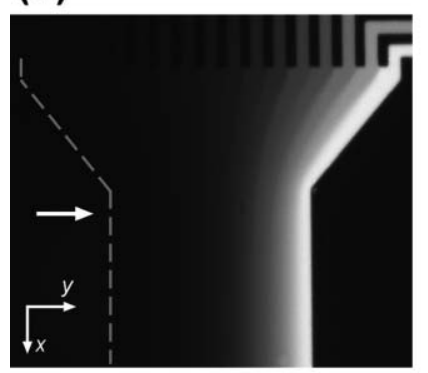

(d)

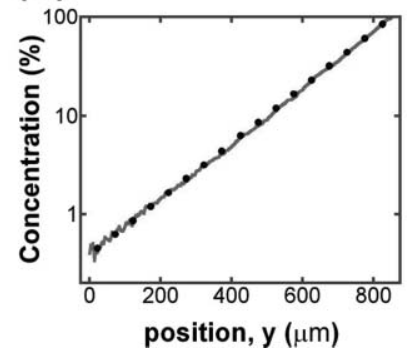

(b)

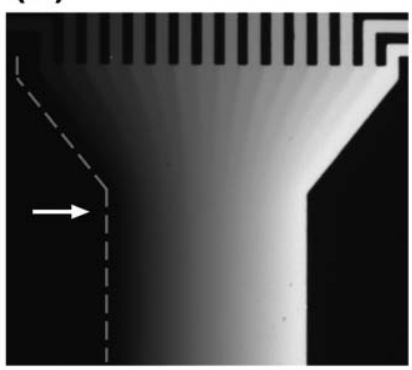

(e)

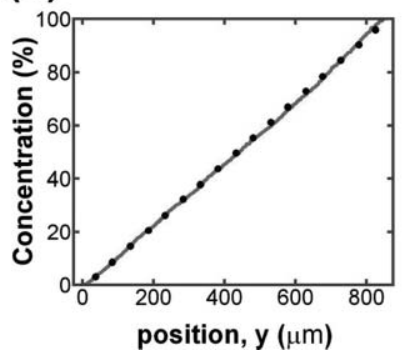

(c)

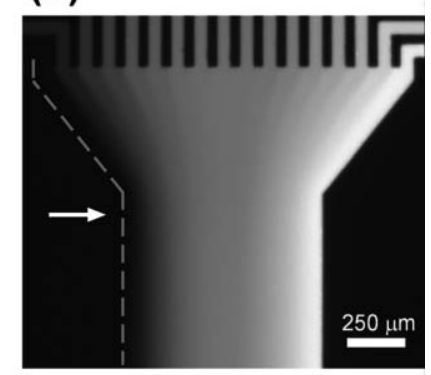

(f)

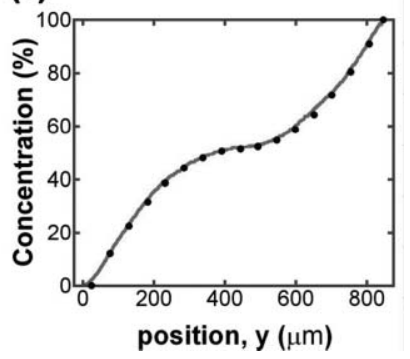

Fig. 4 Distributions of FITC in the test channels of the microfluidic devices. (a), (b) and (c) Fluorescence micrographs of the test channels in the devices generating exponential, linear, and double-parabolic concentration profiles, respectively. Dashed lines are drawn along the left sides of the test channels. (d), (e) and (f) Concentration of FITC as a function of position across the test channel in the devices generating exponential, linear, and double-parabolic concentration profiles, respectively, is shown as a continuous line. The plot in (d) is in semi-logarithmic coordinates. The edges of the test channel correspond to $y=0$ and $850 \mu \mathrm{m}$. The concentration of $100 \%$ corresponds to the solution injected into the inlet 2 , with $15 \mathrm{ppm}$ of FITC. Round black dots in (d)-(f) show fits of the measured concentration profiles to an exponential, linear, and double-parabolic function, respectively. The fitting is made neglecting the data from the $50 \mu \mathrm{m}$ wide regions at the edges. Horizontal arrows in (a)-(c) indicate the locations at which the concentration profiles in (d)-(f) are measured. 
regions with widths on the order of $w_{\mathrm{s}}$ (Fig. 4(d)-(f)). One of the reasons for this difference is the net diffusive mass transfer down the gradient, reducing the concentration at the high concentration edge and increasing it at the low concentration edge. This process occurs concurrently with diffusive smoothening of the discontinuities in concentration between the adjacent streams that have widths $w_{\mathrm{s}}$ and affects regions of similar widths at the margins. In the case of the linear profile (Fig. 4(d)), there are plateaus of zero and maximal concentration of widths of about $w_{c} / 2$ in the marginal regions. A better fit to a straight line would be obtained if the volumetric fluxes of the streams with zero and maximal concentration injected at the edges were reduced by half, $q_{4,1}=q_{4,17}=0.5 q_{4,2-16}$.

Because of relatively high flow velocities, variation of the concentration profiles in the test channels along the flow (in the $x$-direction) was rather slow (Fig. 5). In the case of the exponential profile (Fig. 5(a)), the profile shape was well preserved within the initial $1.5 \mathrm{~mm}$ of the channel (curves 1-3). Degradation of the exponential profile occurred because of the net diffusive transport from the high concentration edge to the low concentration edge and resulted in the development of flattened regions at both edges. Nevertheless, the concentration profile in an internal region between $y=250$ and $700 \mu \mathrm{m}$ remained exponential even at $3.5 \mathrm{~mm}$ from the beginning of the test channel (Fig. 5(a)) with an almost unchanged exponent of $\sim 6.5 \times 10^{-3} \mu^{-1}=\ln (256) / w(w=850 \mu \mathrm{m}$ is the width of the test channel). As expected for an exponential profile, the concentration in this internal region, $c(x, y)$, steadily grew with
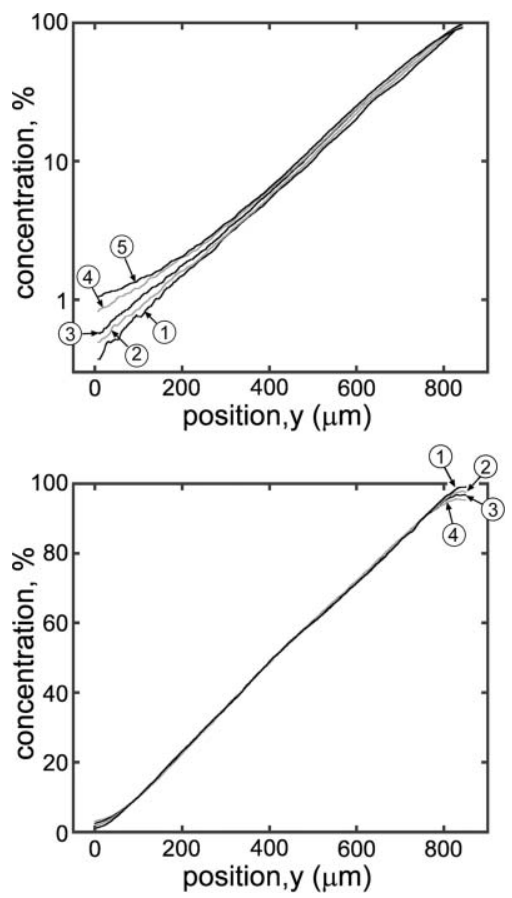

Fig. 5 Concentration of FITC normalized to its maximum concentration as a function of position across the test channel, $c(y)$, at different distances, $x$, from the beginning of the rectilinear part of the test channel. (a) Profiles of concentration in the device generating an exponential profile. Curves $1-5$ correspond to $x=0,0.5,1.5,2.5$, and $3.5 \mathrm{~mm}$. (b) Profiles of concentration in the device generating a linear profile. Curves $1-4$ correspond to $x=0.5,1.5,2.5$, and $3.5 \mathrm{~mm}$. $x$. The increase in concentration over $\Delta x=3 \mathrm{~mm}$ (curve $5 v s$. curve 2 in Fig. 5(a)) was $\sim 15 \%$ on average. This number corresponded to an increase in concentration of only $\sim 0.5 \%$ over $\Delta x=100 \mu \mathrm{m}$, which was more than two orders of magnitude lower than the twofold increase over $\Delta y=100 \mu \mathrm{m}$. The linear concentration profile (Fig. 5(b)) remained practically unchanged over the entire length of the test channel, apart from the development of flattened regions at the edges (with increased concentration at the low concentration edge and decreased concentration at the high concentration edge).

To test the sensitivity of operation of the devices to variations in the inlet pressures, we reduced the pressure at inlet 2 in the devices in Fig. 1(a) and (b) by different amounts, $\Delta P$, and monitored the resulting changes in the concentration profiles. The difference between the concentration profiles was quantified in terms of its maximal and root-mean-square (RMS) values, both normalized to the maximum concentration. For the device generating a linear profile, the maximum and RMS values were $2.1 \%$ and $1.2 \%$ at $\Delta P=125 \mathrm{~Pa}$ and decreased to $1.0 \%$ and $0.5 \%$ at $\Delta P=50 \mathrm{~Pa}$. For the device generating an exponential profile, the maximum and RMS values were $2.7 \%$ and $1.7 \%$ at $\Delta P=125 \mathrm{~Pa}$ and decreased to $1.0 \%$ and $0.8 \%$ at $\Delta P=50 \mathrm{~Pa}$. When extrapolated to a pressure difference of $2 \mathrm{~Pa}$ (the precision of pressure adjustment), both maximum and RMS concentration variations were below $0.1 \%$, suggesting that the devices and the flow control set-up were more than adequate for generating well defined profiles of concentration.

For the device generating the exponential profile, the discharge of liquid through inlets 1 and 2 in $1 \mathrm{~h}$ was $\sim 72 \mu \mathrm{L}$ and $\sim 18 \mu \mathrm{L}$, respectively, corresponding to reductions by $\sim 0.13 \mathrm{~mm}$ and $\sim 0.03 \mathrm{~mm}$ in the levels of liquid in the syringes feeding the two inlets. These liquid level variations were expected to change the ratio between $q_{0,1}$ and $q_{0,2}$ by less than $0.1 \%$, which would have very little influence on the concentration profile. The effect of the liquid discharge was expected to be even smaller for the linear and double parabolic profiles because of equal $q_{0,1}$ and $q_{0,2}$. We tested stability of the concentration profiles in the devices producing linear and exponential profiles by comparing fluorescence micrographs of the same areas of the test channels taken with an interval of $1 \mathrm{hr}$. The root mean square of the difference of the fluorescence intensity between the two profiles was less than $0.5 \%$ and less than $1 \%$ of the maximal fluorescence intensity for the linear and exponential profiles, respectively.

The concentration profiles in Fig. 4 illustrate the versatility of the proposed network architecture. The linear concentration profile has a constant first derivative, $\mathrm{d} c / \mathrm{d} y$, and zero second derivative, $\mathrm{d}^{2} c / \mathrm{d} y^{2}$. In the exponential profile, both derivatives are positive and grow exponentially across the test channel. In the double-parabolic profile, $\mathrm{d} c / \mathrm{d} y$ is linearly decreasing on the left side, linearly increasing on the right side, and $d^{2} c / d y^{2}$ changes its sign in the middle of the test channel. The proposed scheme of building a gradient-making microchannel network can be used to generate any given concentration profile with one essential limitation: $c(y)$ has to be a monotonic function. This limitation originates from the fact that the concentration in any serpentine channel (except for channels at the edges) is a weighted average of the concentrations in the two adjacent 
channels, precluding local minima or maxima of concentration in the networks with two inlets. Concentration profiles with local maxima and minima can be produced by using networks with three or more inlets or by directing streams emerging from several independent networks into a single test channel. ${ }^{6,7}$

With the common planar architecture of the microfluidic devices, each channel can have only one or two channels immediately adjacent to it. Hence, a stream emerging from a channel can be directly merged with only one or two other streams, and by splitting and merging streams from a planar array of $N$ channels, not more than $N-1$ new mixtures can be produced. (This limitation is lifted if a 3D microchannel network is used. ${ }^{30}$ ) Therefore, the growth of the number of distinct concentrations with the number of steps, $N_{k+1}=N_{k}+$ $\left(N_{k}-1\right)$ and $N_{k}=2^{k}+1$, which is realized in the proposed architecture, is the fastest possible under the constraint of a planar network.

The logarithmically small number of steps (stages), $k=$ $\log _{2}\left(N_{k}-1\right)$, needed to generate a given number of distinct concentrations allows significant reduction in area occupied by the network and in pressure required to drive flow through the network compared with the design in ref. 6 and 7. For example, to generate a linear set of 17 concentrations with the same total flow rate, a gradient-making network with the architecture as in ref. 6 and 7 would need to have 15 stages and a net area $\sim 7$ times larger than the network in Fig. 1(b), with $\sim 3$ times higher pressure required to drive the flow in it. To make this comparison, we designed a network with the architecture as in ref. 6 and 7 that had serpentine channels of the same cross-section as in Fig. 1(b) $(50 \times 40 \mu \mathrm{m}$, separated by $50 \mu \mathrm{m}$ ) and horizontal channels of the same width as in Fig. 1(b) $(0.5 \mathrm{~mm})$. One can see that the values of $N$ attainable with the proposed architecture, $N=17$ in the present networks and $N=33$ with just one more stage of splitting and mixing, would be rather impractical with the previous design because of the large footprint of the devices. Large values of $N$ are important for accurate definition of complex continuous profiles $c(y)$ by discrete sets of $c_{n}$ (Fig. 4(b), (c), (e) and (f)) and for stability of $c(y)$ with respect to variations in $x$ and $\bar{v}$.

The gradient-making networks could be further optimized by allowing uneven volumetric fluxes, $q_{n}$, in the serpentine channels of the last stage (which feed into the test channel). In particular, for accurate definition of a profile with large variations of $\mathrm{d} c / \mathrm{d} y$ across the test channel (such as in Fig. 4(c) and (f)), it could be beneficial to have a large number of narrow streams (low $q_{n}$ ) where $\mathrm{d} c / \mathrm{d} y$ is large, and small number of wide streams (high $q_{n}$ ) where $\mathrm{d} c / \mathrm{d} y$ is small. (This would be somewhat analogous to the adaptive mesh used in numeric simulations.) The values of $c_{n}$ would then be selected in accordance with the positions of the streams in the test channel, and the network would be constructed based on $c_{n}$ and $q_{n}$.

\section{Conclusion}

We have described and validated an architecture of microfluidic gradient-making networks, which are more compact and versatile than the networks made using the previous design. A particularly useful feature of the proposed architecture is the ease of generation of exponential concentration profiles that can be applied to studies of chemotaxis and dose response of live cells. In an exponential profile of a chemoattractant, all cells are exposed to the same fractional gradient, $\frac{1}{c} \frac{\mathrm{d} c}{\mathrm{~d} y}$, that remains constant as cells migrate, creating experimental conditions essentially different from those in the linear concentration profile studied before. ${ }^{8-12,14}$ For dose response studies, cells can be presented with solutions containing different concentrations of the substance of interest from the individual serpentine channels at the network outlet. ${ }^{15,16}$ In successive channels of a network generating an exponential profile, the concentrations differ by a constant factor, making it possible to study the dose response in a broad range of concentrations with even fractional resolution, $\Delta c / c$.

\section{Acknowledgements}

The authors are grateful to Virginia Vandelinder for useful suggestions. The work was supported by NSF grant MCB0331306.

\section{References}

1 S. H. Zigmond, J. Cell Biol., 1977, 75, 606-616.

2 D. Zicha, G. A. Dunn and A. F. Brown, J. Cell Sci., 1991, 99, 769-775.

3 H. B. Mao, P. S. Cremer and M. D. Manson, Proc. Natl. Acad. Sci. U. S. A., 2003, 100, 5449-5454.

4 G. M. Walker, M. S. Ozers and D. J. Beebe, Sens. Actuators, B, 2004, 98, 347-355.

5 A. E. Kamholz and P. Yager, Biophys. J., 2001, 80, 155-160.

6 N. L. Jeon, S. K. W. Dertinger, D. T. Chiu, I. S. Choi, A. D. Stroock and G. M. Whitesides, Langmuir, 2000, 16, $8311-8316$

7 S. K. W. Dertinger, D. T. Chiu, N. L. Jeon and G. M. Whitesides, Anal. Chem., 2001, 73, 1240-1246.

8 N. L. Jeon, H. Baskaran, S. K. W. Dertinger, G. M. Whitesides, L. Van de Water and M. Toner, Nat. Biotechnol., 2002, 20, 826-830.

9 F. Lin, C. M. C. Nguyen, S. J. Wang, W. Saadi, S. P. Gross and N. L. Jeon, Biochem. Biophys. Res. Commun., 2004, 319, 576-581.

10 F. Lin, C. M. C. Nguyen, S. J. Wang, W. Saadi, S. P. Gross and N. L. Jeon, Ann. Biomed. Eng., 2005, 33, 475-482.

11 G. M. Walker, J. Q. Sai, A. Richmond, M. Stremler, C. Y. Chung and J. P. Wikswo, Lab Chip, 2005, 5, 611-618.

12 D. Irimia, S. Y. Liu, W. G. Tharp, A. Samadani, M. Toner and M. C. Poznansky, Lab Chip, 2006, 6, 191-198.

13 S. J. Wang, W. Saadi, F. Lin, C. M. C. Nguyen and N. L. Jeon, Exp. Cell Res., 2004, 300, 180-189.

14 L. L. Song, S. M. Nadkarni, H. U. Bodeker, C. Beta, A. Bae, C. Franck, W. J. Rappel, W. F. Loomis and E. Bodenschatz, Eur. J. Cell Biol., 2006, 85, 981-989.

15 D. M. Thompson, K. R. King, K. J. Wieder, M. Toner, M. L. Yarmush and A. Jayaraman, Anal. Chem., 2004, 76, 4098-4103.

16 P. J. Hung, P. J. Lee, P. Sabounchi, R. Lin and L. P. Lee, Biotechnol. Bioeng., 2005, 89, 1-8.

17 J. Pihl, J. Sinclair, E. Sahlin, M. Karlsson, F. Petterson, J. Olofsson and O. Orwar, Anal. Chem., 2005, 77, 3897-3903.

18 R. Ferrigno, J. N. Lee, X. Y. Jiang and G. M. Whitesides, Anal. Chem., 2004, 76, 2273-2280.

19 S. K. W. Dertinger, X. Y. Jiang, Z. Y. Li, V. N. Murthy and G. M. Whitesides, Proc. Natl. Acad. Sci. U. S. A., 2002, 99, $12542-12547$.

20 X. Y. Jiang, Q. B. Xu, S. K. W. Dertinger, A. D. Stroock, T. M. Fu and G. M. Whitesides, Anal. Chem., 2005, 77, 2338-2347.

21 R. C. Gunawan, J. Silvestre, H. R. Gaskins, P. J. A. Kenis and D. E. Leckband, Langmuir, 2006, 2006. 
22 N. Zaari, P. Rajagopalan, S. K. Kim, A. J. Engler and J. Y. Wong, Adv. Mater., 2004, 16, 2133.

23 J. A. Burdick, A. Khademhosseini and R. Langer, Langmuir, 2004, 20, 5153-5156.

24 U. Levy, K. Campbell, A. Groisman, S. Mookherjea and Y. Fainman, Appl. Phys. Lett., 2006, 88, Art. \# 111107.

25 F. Lin, W. Saadi, S. W. Rhee, S. J. Wang, S. Mittal and N. L. Jeon, Lab Chip, 2004, 4, 164-167.
26 J. Sager, M. Young and D. Stefanovic, Langmuir, 2006.

27 D. Irimia, D. A. Geba and M. Toner, Anal. Chem., 2006, 78, 3472-3477.

28 A. Groisman, M. Enzelberger and S. R. Quake, Science, 2003, 300, 955-958.

29 F. M. White, Fluid Mechanics, McGraw-Hill, New York, 2002.

30 C. Neils, Z. Tyree, B. Finlayson and A. Folch, Lab Chip, 2004, 4, $342-350$.

\section{Find a SOLUTION}

\section{... with books from the RSC}

\section{Choose from exciting textbooks, research level books or reference books in a wide range of subject areas, including:}

- Biological science

- Food and nutrition

- Materials and nanoscience

- Analytical and environmental sciences

- Organic, inorganic and physical chemistry

\section{Look out for $\mathbf{3}$ new series coming soon ...}

- RSC Nanoscience \& Nanotechnology Series

- Issues in Toxicology

- RSC Biomolecular Sciences Series

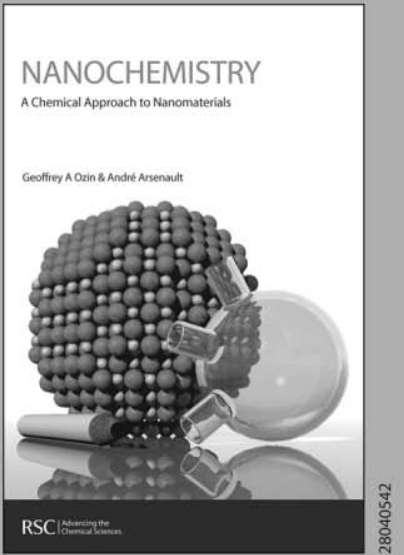

